\title{
TOPOLOGICAL PROPERTIES OF PARANORMAL OPERATORS ON HILBERT SPACE
}

BY

\author{
GLENN R. LUECKE
}

\begin{abstract}
Let $B(H)$ be the set of all bounded endomorphisms (operators) on the complex Hilbert space $H . T \in B(H)$ is paranormal if $\left\|(T-z I)^{-1}\right\|=$ $1 / d(z, \sigma(T))$ for all $z \notin \sigma(T)$ where $d(z, \sigma(T))$ is the distance from $z$ to $\sigma(T)$, the spectrum of $T$. If $\mathscr{P}$ is the set of all paranormal operators on $H$, then $\mathscr{P}$ conta ins the normal operators, $\mathcal{X}$, and the hyponormal operators; and $\mathcal{P}$ is contained in $\mathcal{L}$, the set of all $T \in B(H)$ such that the convex hull of $\sigma(T)$ equals the closure of the numerical range of $T$. Thus, $\Re \subseteq \mathscr{P} \subseteq \mathfrak{L} \subseteq B(H)$. Give $B(H)$ the norm topology. The main results in this paper are $(1) \mathcal{\eta}, \mathcal{S}$, and $\mathcal{Q}$ are nowhere dense subsets of $B(H)$ when $\operatorname{dim} H \geq 2,(2) \Re, \mathfrak{P}$, and $\mathcal{Q}$ are arcwise connected and closed, and (3) $\mathcal{Y}$ is a nowhere dense subset of $\mathcal{P}$ when $\operatorname{dim} H$ $=\infty$.
\end{abstract}

Paranormal operators have received considerable attention in the current literature ([16], [17], [19], [20], [21], [23], [24], [25]). However, only the various spectral properties of paranormal operators have been discussed. In this paper, the topological properties of the set of all paranormal operators $\mathcal{P}$ on a Hilbert space $H$ are investigated.

We begin by giving the notation to be used and by defining some of the more specialized terminology. The point spectrum and approximate point spectrum of an operator $T$ are denoted by $\sigma_{p}(T)$ and $\sigma_{\pi}(T)$, respectively. $z \in \sigma_{p}(T)$ is a normal eigenvalue if $\{x \in H:(T-z I) x=0\}=\left\{x \in H:(T-z I)^{*} x=0\right\}$ where $I$ denotes the identity operator on $H . z \in \sigma_{\pi}(T)$ is a normal approximate eigenvalue of $T$ when (1) $\left\|x_{n}\right\|=1$ and $\left\|(T-z I) x_{n}\right\| \rightarrow 0$ as $n \rightarrow \infty$ imply $\left\|(T-z I)^{*} x_{n}\right\| \rightarrow 0$ as $n \rightarrow \infty$, and (2) $\left\|y_{n}\right\|=1$ and $\left\|(T-z I)^{*} y_{n}\right\| \rightarrow 0$ as $n \rightarrow \infty$ imply $\left\|(T-z I) y_{n}\right\| \rightarrow 0$ as $n \rightarrow \infty$. If $S$ is a set of complex numbers, then $\partial S$ denotes the boundary of $S$ and $\operatorname{co}(S)$ denotes the convex hull of $S$. Let $W(T)=\operatorname{closure}\{(T x, x): x \in H,\|x\|=1\}$ denote the closure of the numerical range of $T$. Let $R(T, z)=(T-z I)^{-1}$ for each $z \in \rho(T)$, the resolvent set of $T$. The spectral radius of $T$ is denoted by $R_{\mathrm{sp}}(T)$. $T \in B(H)$ is hyponormal if $T^{*} T-T T^{*} \geq 0$.

I. Elementary properties of paranormal operators. Stampfli [19] has shown that every hyponormal operator is paranormal. Therefore, since every normal

Presented to the Society, August 28, 1970; received by the editors April 16, 1970. AMS (MOS) subject classifications (1969). Primary 4740.

Key words and phrases. Hilbert space, resolvent, normal operator, convexoid operators, properties and topological properties of $G_{1}$ operators. 
operator is hyponormal, $\Re \subseteq \mathfrak{P}$. G. Orland [13] proved that $T \in \mathscr{Q}$ if and only if $\|R(T, z)\| \leq 1 / d(z$, co $\sigma(T))$ for all $z \notin \operatorname{co} \sigma(T)$. From this it follows immediately that if $T$ is a paranormal operator, then co $\sigma(T)=W(T)$, i.e. $\mathfrak{P} \subseteq \mathcal{Q}$.

Theorem 1.1. If $T$ is a paranormal operator and if $\alpha$ and $\beta$ are complex numbers, then $\alpha T+\beta I$ and $T^{*}$ are paranormal.

The proof is a simple computation that depends on $\sigma(\alpha T+\beta I)=\alpha \sigma(T)+\beta$ and $\sigma\left(T^{*}\right)=\sigma(T)^{*}$.

The following theorem is very useful in constructing examples.

Theorem 1.2. If $A$ is any operator on $H$, then there exists a Hilbert space $K$ and a normal operator $N$ on $K$ sucb that $T=A \oplus N \in B(H \oplus K)$ is paranormal.

Proof. Since $W(A)$ is a compact set of complex numbers, there exists a Hilbert space $K$ and a normal operator $N$ on $K$ such that $\sigma(N)=W(A)$ [3, p. 581]. Let $T=A \oplus N$. Then $\sigma(T)=\sigma(N)=W(A)$. For $z \notin \sigma(T)=W(A)$, it is well known that $\|R(A, z)\| \leq 1 / d(z, W(A))=1 / d(z, \sigma(T))$. Since $N$ is normal and $z \in \rho(N)$, $\|R(N, z)\|=1 / d(z, \sigma(T))$. Therefore

$$
\|R(T, z)\|=\operatorname{Max}\{\|R(A, z)\|,\|R(N, z)\|\}=1 / d(z, \sigma(T)) .
$$

Thus $T$ is paranormal.

As we shall see, the class of all hyponormal operators on $H$ is distinct, in general, from the class $\mathcal{P}$ of paranormal operators. We know [19] that if $T$ is hyponormal, then $\|T\|=R_{\mathrm{sp}}(T)$ and if $T$ is also invertible, then $T^{-1}$ is hyponormal. These properties do not generalize to paranormal operators.

Theorem 1.3. There exists an invertible paranormal operator $T$ such that

1. $T$ is not byponormal,

2. $T^{2}$ is not paranormal,

3. $\|T\|>R_{\mathrm{sp}}(T)$, and

4. $T^{-1}$ is not paranormal.

Proof. Let $A=\left[\begin{array}{ll}1 & 1 \\ 0 & 1\end{array}\right]$. Let $N$ be a normal operator such that $\sigma(N)=W(A)$, and let $T=A \oplus N$. Then by Theorem 1.2, $T$ is paranormal. We know from [19] that a hyponormal operator is hyponormal on invariant subspaces. Therefore, since $A$ is not hyponormal, $T$ is not hyponormal. By [1], $W(A)$ is the closed disc of radius $1 / 2$ about $z=1$, and $W\left(A^{2}\right)$ is the closed disc of radius 1 about $z=1$. Therefore

$$
0 \in W\left(A^{2}\right) \subseteq W\left(T^{2}\right) \text { and } \quad 0 \notin \operatorname{co}\left(\sigma(T)^{2}\right)=\operatorname{co} \sigma\left(T^{2}\right) .
$$

Therefore, $\operatorname{co} \sigma\left(T^{2}\right) \neq W\left(T^{2}\right)$ and so $T^{2}$ is not paranormal. Let $x=\left[\begin{array}{l}\sqrt{1} / 2 \\ \sqrt{1} / 2\end{array}\right]$, then $\|x\|=1$ and $\|A x\|=\sqrt{10} / 2$. Then

$$
\|T\| \geq\|A x\|=\sqrt{10} / 2>3 / 2=R_{\mathrm{sp}}(T) .
$$


Therefore $\|T\|>R_{\mathrm{sp}}(T)$. If $T^{-1}$ were paranormal, then

$$
\|T\|=\left\|R\left(T^{-1}, 0\right)\right\|=1 / d\left(0, \sigma\left(T^{-1}\right)\right)=R_{\mathrm{sp}}(T) .
$$

Contradiction. Hence $T^{-1}$ is not paranormal.

II. Topological properties of $\mathscr{P}$. Recall that $\Re$ is the set of all normal operators on $H, \mathcal{P}$ is the set of all paranormal operators on $H, \mathcal{Q}$ is the set of all $T \in B(H)$ such that co $\sigma(T)=W(T)$, and $\Re \subseteq \mathcal{P} \subseteq \mathcal{Q}$. It will always be assumed that $B(H)$ has the uniform operator (norm) topology.

The following notation will be used in this section: If $S$ is a compact subset of the complex numbers $\mathbf{C}$ and if $\epsilon>0$, then let $S+(\epsilon)=\{z: d(z, S)<\epsilon\}$. If $S$ and $S_{n}, n=1,2,3, \cdots$, are compact sets in $\mathbf{C}$, then the sequence $\left\{S_{n}\right\}$ approaches $S$, written $S_{n} \rightarrow S$, if for every $\epsilon>0$ there exists a positive integer $N$ such that, for $n \geq N, S_{n} \subseteq S+(\epsilon)$ and $S \subseteq S_{n}+(\epsilon)$.

In general $\sigma(T)$ is not a continuous function of $T$ in $B(H)$ (see [7, problem 85]), but $\sigma(T)$ is continuous if we restrict $T$ to $\mathcal{P}$.

Theorem 2.1. If $\left\{T_{n}\right\}$ is a sequence of paranormal operators approaching the operator $T$ in norm, then $\sigma\left(T_{n}\right) \rightarrow \sigma(T)$ as $n \rightarrow \infty$.

To prove this theorem we need the following lemma from [7, problem 86].

Lemma. If $T \in B(H)$ and $\epsilon>0$, then there exists $\delta>0$ sucb that if $S \in B(H)$ and $\|T-S\|<\delta$, then $\sigma(S) \subseteq \sigma(T)+(\epsilon)$.

Proof of Theorem 2.1. We know by the lemma that for each $\epsilon>0$ there exists a positive integer $N$ such that, for $n \geq N, \sigma\left(T_{n}\right) \subseteq \sigma(T)+(\epsilon)$. Therefore, to show $\sigma\left(T_{n}\right) \rightarrow \sigma(T)$, it suffices to show that for each $\epsilon>0$ there exists a positive integer $N$ such that $\sigma(T) \subseteq \sigma\left(T_{n}\right)+(\epsilon)$ for all $n \geq N$. If this does not hold, then without loss of generality we may assume that there exists $\epsilon>0$ and a sequence $\left\{z_{n}\right\}$ $\subseteq \sigma(T)$ such that $d\left(z_{n}, \sigma\left(T_{n}\right)\right) \geq \epsilon$ for all $n$. Since $\sigma(T)$ is compact, we may assume $z_{n} \rightarrow z \in \sigma(T)$. If $\left|z_{n}-z\right|<\epsilon / 2$, then

$$
d\left(z, \sigma\left(T_{n}\right)\right) \geq d\left(z_{n}, \sigma\left(T_{n}\right)\right)-\left|z-z_{n}\right| \geq \epsilon-\epsilon / 2=\epsilon / 2 .
$$

Hence

$$
\left\|R\left(T_{n}, z\right)\right\|=1 / d\left(z, \sigma\left(T_{n}\right)\right) \leq 2 / \epsilon .
$$

Now choose $m$ so that $\left\|\left(T_{m}-T\right) R\left(T_{m}, z\right)\right\|<1$, then $l-\left(T_{m}-T\right) R\left(T_{m}, z\right)$ is invertible [7, problem 173]. Let

$$
A=R\left(T_{m}, z\right)\left(I-\left(T_{m}-T\right) R\left(T_{m}, z\right)\right)^{-1} .
$$

Then $A(T-z I)=(T-z I) A=I$ so that $z \in \rho(T)$. Contradiction.

Remark. Note that the full strength of the paranormality assumption was not 
used in this proof, and that one can easily devise various larger classes of operators on which $\sigma(T)$ is continuous.

Theorem 2.2. $\mathcal{P}$ is an arcwise connected, closed subset of $B(H)$.

Proof. Since $T \in \mathscr{P}$ implies $a T \in \mathcal{P}$ for every complex number $a$, we see that the ray in $B(H)$ through $T$ is contained in $\mathscr{P}$. Therefore $\mathscr{P}$ is arcwise connected.

Suppose $T_{n} \rightarrow T,\left\{T_{n}\right\}$ a sequence of operators in $\mathcal{P}$, and $T \in B(H)$. Let $z \epsilon$ $\rho(T)$. By the lemma to Theorem 2.1,

$$
\limsup _{n \rightarrow \infty} \frac{1}{d\left(z, \sigma\left(T_{n}\right)\right)} \leq \frac{1}{d(z, \sigma(T))}
$$

Therefore, since $\left\|R\left(T_{n}, z\right)\right\|=1 / d\left(z, \sigma\left(T_{n}\right)\right)$ whenever $z \in \rho\left(T_{n}\right)$, there exists a positive integer $N$ such that the sequence $\left\{\left\|R\left(T_{n}, z\right)\right\|: n \geq N\right\}$ is bounded. Then, since $R(T, z)-R\left(T_{n}, z\right)=R(T, z)\left(T-T_{n}\right) R\left(T_{n}, z\right),\left\|R\left(T_{n}, z\right)\right\| \rightarrow\|R(T, z)\|$ as $n \rightarrow \infty$. Consequently,

$$
\|R(T, z)\|=\lim _{n \rightarrow \infty}\left\|R\left(T_{n^{\prime}} z\right)\right\|=\lim _{n \rightarrow \infty} \frac{1}{d\left(z, \sigma\left(T_{n}\right)\right)} \leq \frac{1}{d(z, \sigma(T))} .
$$

Since the general $\|R(T, z)\| \geq 1 / d(z, \sigma(T)), T$ is paranormal.

Theorem 2.3. $\mathcal{L}$ is an arcwise connected, closed subset of $B(H)$.

Proof. Since $T \in \mathcal{L}$ implies that $a T \in \mathcal{Q}$ for every complex number $a, \mathfrak{L}$ is arcwise connected.

Let $T_{n} \rightarrow T,\left\{T_{n}\right\} \subseteq \mathcal{Q}$ and $T \in B(H)$. Since $\left|\left(T_{n} x, x\right)-(T x, x)\right| \leq\left\|T_{n}-T\right\|$ for $\|x\|=1, W\left(T_{n}\right) \subseteq W(T)+\left(2\left\|T-T_{n}\right\|\right)$ and $W(T) \subseteq W\left(T_{n}\right)+\left(2\left\|T-T_{n}\right\|\right)$. Consequently, $W\left(T_{n}\right) \rightarrow W(T)$. Let $\epsilon>0$, then by the lemma to Theorem 2.1 there exists a positive integer $N$ such that $\sigma\left(T_{n}\right) \subseteq \sigma(T)+(\epsilon)$ for all $n \geq N$. Therefore, for $n \geq N$, co $\sigma\left(T_{n}\right) \subseteq \operatorname{co} \sigma(T)+(\epsilon)$ and hence

$$
W(T)=\lim _{n \rightarrow \infty} W\left(T_{n}\right)=\lim _{n \rightarrow \infty} \operatorname{co} \sigma\left(T_{n}\right) \subseteq \operatorname{co} \sigma(T)+(\epsilon) .
$$

Since $\epsilon>0$ is arbitrary, $W(T) \subseteq$ co $\sigma(T)$. Since in general co $\sigma(T) \subseteq W(T), T \in \mathfrak{L}$.

Let $\pi$ be the set of all normal operators on $H$. Since $\left\|T_{n}-T\right\| \rightarrow 0$ implies $\left\|T_{n}^{*}-T^{*}\right\| \rightarrow 0, \Re$ is closed in the uniform operator topology on $B(H)$. Since $T \in \Re$ implies $a T \in \Re$ for any complex $a, \pi$ is arcwise connected.

We already know that $\Re \subseteq P \subseteq \mathscr{P} \subseteq B(H)$. We will now investigate the relative topological properties of these four sets. Stampfli [20, Theorem C] has shown that $\pi=\mathscr{P}$ when $\operatorname{dim} H<\infty$. However, when $\operatorname{dim} H=\infty$, then $\pi$ is a very "thin" subset of $\mathcal{P}$.

Theorem 2.4. $\pi$ is a nowbere dense subset of $\mathcal{P}$ when $\operatorname{dim} H=\infty$.

Proof. Since $\pi$ is closed, to show that $\pi$ is a nowhere dense subset of $\mathcal{P}$, 
it suffices to show that $\Re$ has empty interior in $\mathcal{P}$. Let $T \in \Re$ and let $\epsilon>0$.

First suppose that $T$ has an eigenvalue of infinite multiplicity. We may assume that the eigenvalue is zero. Let $M$ be the eigenspace of zero. Then $\operatorname{dim} M=$ $\infty$, $M$ reduces $T$, and we can write $T=B \oplus Z$ where $Z$ is the zero operator on $M$. Let $S=\left[\begin{array}{ll}0 & \epsilon \\ 0 & 0\end{array}\right] \oplus N$ be a nonnormal paranormal operator [see Theorem 1.2] on $M$ with $N$ a normal operator such that $\sigma(N)=W\left[\begin{array}{ll}0 & \epsilon \\ 0 & 0\end{array}\right]$. Then $B \oplus S$ is a nonnormal paranormal operator such that $\|T-B \oplus S\|=\|B \oplus Z-B \oplus S\|=\|S\|=\epsilon$. The last equality holds since $\|N\|=R_{\mathrm{sp}}(N)=\epsilon / 2$ and $\left\|\left[\begin{array}{ll}0 & \epsilon \\ 0 & 0\end{array}\right]\right\|=\epsilon$. Therefore, since $\epsilon>0$ is arbitrary, $T$ is not contained in the interior of $\pi$ in $\mathcal{P}$.

If $\sigma(T)$ is finite and $T \in \Re$, then $\sigma(T)=\sigma_{p}(T)$ and $T$ has an eigenvalue of infinite multiplicity. We therefore assume that $\sigma(T)$ is infinite and that zero is an accumulation point of $\sigma(T)$. Let $D$ be the open disc about zero of radius $\epsilon / 2$. Let $E$ be the resolution of the identity for $T$ so that $T=\int_{\sigma(T)} z d E_{z}$. Let $M=$ $(E(D))(H), P=\sigma(T)-D$, and let $A=\int_{P} z d E_{z}$. Then $M$ reduces $T, \operatorname{dim} M=\infty$, and $A$ is a normal operator. Let $Z$ be the zero operator on $M$. Then $A \oplus Z$ is a normal operator with zero an eigenvalue of infinite multiplicity, and

$$
\|T-A \oplus Z\|=\left\|\int_{D} z d E_{z}\right\| \leq \epsilon / 2
$$

By the first part of this proof, there exists a nonnormal paranormal operator $S$ such that $\|A \oplus Z-S\|<\epsilon / 2$. Then

$$
\|T-S\| \leq\|T-A \oplus Z\|+\|A \oplus Z-S\|<\epsilon .
$$

Therefore, since $\epsilon>0$ is arbitrary, $T$ is not contained in the interior of $\mathcal{N}$ in $\mathcal{P}$. Hence the interior of $\pi$ in $\mathscr{P}$ is empty.

Define $C_{2}$ to be the set of all operators $T \in \mathscr{L}$ with $W(T)$ a closed line segment or a point. For $k=3,4,5, \cdots$, let $C_{k}$ be the set of all operators $T \in \mathscr{L}$ such that $W(T)$ is the convex hull of a nondegenerate polygon with $k$ sides. If $T \in C_{k}$, then each vertex of $W(T)$ must be in the spectrum of $T$ [1]. S. Hildebrandt [10, Theorem 2] has shown that, if $z \in \sigma_{p}(T) \cap \partial W(T)$, then $z$ is a normal eigenvalue of $T$. Thus for $T \in C_{k}$, the vertices of $W(T)$ are normal eigenvalues of $T$, when $\operatorname{dim} H<\infty$. Hence, all the operators in $C_{n} \cup C_{n-1}$ are normal operators when $\operatorname{dim} H=n<\infty$.

In [10, Theorem 9] S. Hildebrandt showed that $\Re=\mathscr{P}=\mathscr{L}$ when $\operatorname{dim} H \leq 4$, and that $\pi \neq \mathscr{L}$ for $5 \leq \operatorname{dim} H<\infty$. The following theorem gives more information on how $\mathcal{P}$ and $\mathcal{L}$ are related when $5 \leq \operatorname{dim} H<\infty$. Recall that $\Re=\mathscr{P}$ for $\operatorname{dim} H<\infty$.

Theorem 2.5. If $5 \leq \operatorname{dim} H=n<\infty$, then the interior of $\mathcal{P}$ in $\mathcal{Q}$ equals $C_{n} \cup$ $C_{n-1}$.

Proof. Suppose $T \in C_{n} \cup C_{n-1}$. Since $C_{n} \cup C_{n-1} \subseteq \pi, T$ is normal. First, we show that there exists $\epsilon>0$ such that whenever $S \epsilon \mathfrak{L}$ and $\|T-S\|<\epsilon$, then 
$S \in C_{n} \cup C_{n-1}$. To show this, suppose the statement were false. Then there would exist $\left\{S_{n}\right\} \subseteq \mathfrak{Q}$ such that $\left\|T-S_{n}\right\| \rightarrow 0$ and $S_{n} \in \bigcup_{i=2}^{n-2} C_{i}$. Then, since $W\left(S_{n}\right) \rightarrow W(T), T \in \bigcup_{i=2}^{n-2} C_{i}$. Contradiction. Hence, $T$ is an interior point of $P$ in $\mathcal{E}$.

Let $T$ be contained in the interior of $\mathcal{P}=\pi$ in $\mathcal{L}$. Suppose $T \notin C_{n} \cup C_{n-1}$. Let $\epsilon>0$. Since $\operatorname{co} \sigma(T)=W(T), T \in C_{k}$, for some $k \leq n-2$. Since $\operatorname{dim} H \geq 5$ and since $T$ is a normal operator in $C_{k}$, there exists a normal operator $N$ such that

1. $\|T-N\|<\epsilon / 2$,

2. $W(N)$ is a polygon with at least three sides, and

3. $N$ has at least two eigenvalues $z, w$ contained in the interior of $W(N)$.

Write $N=A \oplus B$ where $B$ can be written as $B=\left[\begin{array}{cc}z & 0 \\ 0 & w\end{array}\right]$. Let $a>0$ and let $C=\left[\begin{array}{cc}z & a \\ 0 & w\end{array}\right]$; then $\|B-C\|=\left\|\left[\begin{array}{cc}0 & a \\ 0 & 0\end{array}\right]\right\|=a$. Choose $a>0$ small enough so that $W(C) \subseteq$ $W(N)$ and so that $a<\epsilon / 2$. Then since $W(A)=W(N)$ and $\sigma(A \oplus C)=\sigma(N)$, $\operatorname{co} \sigma(A \oplus C)=W(A \oplus C)$. Hence $A \oplus C \in \mathfrak{L}$. Since $A \oplus C$ is not normal, and since

$$
\|T-A \oplus C\| \leq\|T-N\|+\|N-A \oplus C\| \leq \epsilon / 2+\epsilon / 2=\epsilon,
$$

$T$ is not an interior point of $\mathcal{P}$ in $\mathscr{\mathcal { C }}$. Contradiction. Hence $T \in C_{n} \cup C_{n-1}$.

It is an open question as to what the interior of $\mathcal{P}$ in $\mathscr{L}$ is when $\operatorname{dim} H=\infty$. However, it can be shown that $\mathcal{P} \neq \mathfrak{Q}$ when $\operatorname{dim} H=\infty$.

Theorem 2.6. $\mathfrak{P} \neq \mathfrak{Q}$ when $\operatorname{dim} H=\infty$.

Proof. Write $H=M \oplus M^{\perp}$ where $\operatorname{dim} M=5$. Let

$$
A=\left[\begin{array}{ll}
0 & 1 \\
0 & 0
\end{array}\right] \text { and } N=\left[\begin{array}{lll}
a & 0 & 0 \\
0 & b & 0 \\
0 & 0 & c
\end{array}\right]
$$

where $a, b, c$ are three complex numbers that form a triangle with $W(A)$ contained in the interior of the triangle. Consider $A \oplus N$ as an operator on $M$ and observe that $\operatorname{co} \sigma(A \oplus N)=W(N)=W(A \oplus N)$. Since $A \oplus N$ is not normal and since $\operatorname{dim} M<\infty, A \oplus N$ is not paranormal. Hence there exists $z \in \rho(A \oplus N)$ such that

$$
\|R(A \oplus N, z)\|>1 / d(z, \sigma(A \oplus N)) .
$$

Let $I$ be the identity operator on $M^{\perp}$ and let $T=A \oplus N \oplus a I$. Then, $\sigma(T)=$ $\sigma(A \oplus N)$ and $W(T)=W(A \oplus N)$. Therefore $T \in \mathfrak{L}$. Since $d(z, \sigma(T)) \leq|z-a|$,

$$
\begin{aligned}
\|R(T, z)\| & =\operatorname{Max}\left\{\|R(A \oplus N, z)\|,|z-a|^{-1}\right\} \\
& =\|R(A \oplus N, z)\|>1 / d(z, \sigma(T)) .
\end{aligned}
$$

Therefore $T$ is not paranormal.

We now show that if $\operatorname{dim} H \geq 2$, then $\mathcal{L}$ is a nowhere dense ("thin") subset of $B(H)$. Once this is shown, it follows immediately that $\Re$ and $\mathcal{P}$ are also nowhere dense subsets of $B(H)$.

Theorem 2.7. $\mathcal{L}$ is a nowbere dense subset of $B(H)$ when $\operatorname{dim} H \geq 2$. 
To prove this theorem we need the following two technical lemmas.

Lemma 1. If $z_{1}$ and $z_{2}$ are distinct, normal approximate eigenvalues of $T \epsilon$ $B(H)$, then there exist sequences $\left\{x_{n}\right\}$ and $\left\{y_{n}\right\}$ of unit vectors in $H$ such that

1. $\left(x_{n}, y_{n}\right)=0$ for all $n$,

2. $\left\|\left(T-z_{1} I\right) x_{n}\right\| \rightarrow 0$ as $n \rightarrow \infty$, and

3. $\left\|\left(T-z_{2} I\right) y_{n}\right\| \rightarrow 0$ as $n \rightarrow \infty$.

Lemma 2. If $T \in \mathfrak{L}$ such that there exists distinct $a, b \in \partial W(T) \cap \sigma_{p}(T)$, then $T$ is not contained in the interior of $\mathfrak{Q}$.

Proof of Theorem 2.7. Since $\mathfrak{Q}$ is closed (Theorem 2.3), to show that $\mathscr{L}$ is nowhere dense it suffices to show that $\mathscr{Q}$ has empty interior.

We first show that if $T$ is in the interior of $\mathfrak{Q}$, then $\sigma(T)$ must contain at least two points. Suppose $T \in \mathfrak{L}$ and $\sigma(T)=\{a\}$. Then $((T-a I) x, x)=0$ for all $x \in H$ so that $T=a l$. Since $\operatorname{dim} H \geq 2$, write $H=M \oplus M^{\perp}$ where $\operatorname{dim} M=2$. Let $b>0$ and define $A \in B(H)$ as

$$
A=\left[\begin{array}{ll}
0 & b \\
0 & 0
\end{array}\right] \text { on } M, \quad \text { and } A=0 \text { on } M^{\perp} .
$$

Then $\sigma(T+A)=\{a\}$ and, since $b \neq 0,\{a\} \neq W(T+A)$. Therefore $T+A \notin \mathcal{L}$. Since $\|A\|=b>0$ is arbitrary, $T \notin$ interior $\mathscr{L}$.

With the above remark completed, we can now finish the proof of Theorem 2.7. Suppose the theorem were false and there exists $T \in$ interior $\mathcal{L}$. Then there exists $\epsilon>0$ such that whenever $V \in B(H)$ and $\|T-V\|<\epsilon$, then $V \in \mathscr{L}$. From the above remark $\sigma(T)$ must contain at least two points. There must be at least two extreme points of $W(T)$, since extreme points of $W(T)$ for $T \in \mathscr{L}$ are extreme points of $\sigma(T)$. Hence, after a rotation, if necessary, we may assume there exists $z_{1}$, $z_{2} \in \sigma_{\pi}(T) \cap \partial W(T)$ such that

1. $\operatorname{Re} z_{1}=\inf \operatorname{Re} W(T)$,

2. $\operatorname{Re} z_{2}=\sup \operatorname{Re} W(T)$, and

3. $\operatorname{Re} z_{1}<\operatorname{Re} z_{2}$.

Since $z_{1}, z_{2} \in \partial W(T) \cap \sigma_{\pi}(T), z_{1}$ and $z_{2}$ are normal approximate eigenvalues of $T$ [10, Theorem 2, p. 233]. By Lemma 1 there exist unit vectors $x, y \in H$ such that $(x, y)=0,\left\|\left(T-z_{1} I\right) x\right\|<\epsilon / 8$, and $\left\|\left(T-z_{2} I\right) y\right\|<\epsilon / 8$. Let $M$ be the closed subspace spanned by $\{x, y\}$. Define $C \in B(H)$ as

$$
\begin{aligned}
& C x=-(\epsilon / 4) x, \\
& C y=+(\epsilon / 4) y, \text { and } \\
& C z=0 \text { for all } z \in M^{\perp} .
\end{aligned}
$$

Since $\|C\| \leq \epsilon / 2, T+C \in \mathscr{L}$. Since

$$
\begin{aligned}
((T+C) x, x) & =(T x, x)-\epsilon / 4 \text { and } \\
\left|(T x, x)-z_{1}\right| & \leq\left\|\left(T-z_{1} I\right) x\right\|<\epsilon / 8,
\end{aligned}
$$


we obtain inf $\operatorname{Re} W(T+C)<\operatorname{Re} z_{1}$. Since $T+C \in \mathscr{L}$, there exists $a \in \sigma_{\pi}(T+C) \cap$ $\partial W(T+C)$ such that $\operatorname{Re} a=\inf \operatorname{Re} W(T+C)$. Since $C$ is a compact operator, Weyl's spectral inclusion theorem [7, problem 143] yields $\sigma(T+C)-\sigma_{p}(T+C) \subseteq \sigma(T)$. Therefore, $a \in \sigma_{p}(T+C)$ so that $a \in \sigma_{p}(T+C) \cap \partial W(T+C)$. Similarly one shows there exists

$$
b \in \sigma_{p}(T+C) \cap \partial W(T+C)
$$

such that $\operatorname{Re} b=\sup W(T+C)>\operatorname{Re} z_{2}$, and hence $a \neq b$. By Lemma 2, there exists $S \in B(H)$ such that $\|S\|<\epsilon / 2$ and $T+C+S \notin \mathcal{L}$. However, $\|T-(T+C+S)\| \leq$ $\|C\|+\|S\|<\epsilon$ and so by assumption $T+C+S \in \mathcal{L}$. Contradiction.

Proof of Lemma 1. There exist sequences $\left\{w_{n}\right\}$ and $\left\{y_{n}\right\}$ of unit vectors in $H$ such that $\left\|\left(T-z_{1} I\right) w_{n}\right\| \rightarrow 0$ and $\left\|\left(T-z_{2} I\right) y_{n}\right\| \rightarrow 0$ as $n \rightarrow \infty$. Then

$$
\begin{aligned}
& \left|\left(z_{1}-z_{2}\right)\left(w_{n}, y_{n}\right)\right|=\left|\left(z_{1} w_{n}, y_{n}\right)-\left(w_{n}, z_{2}^{*} y_{n}\right)\right| \\
& \quad \leq\left|\left(\left(T-z_{1} I\right) w_{n}, y_{n}\right)\right|+\left|\left(w_{n},\left(T-z_{2} I\right)^{*} y_{n}\right)\right| \leq\left\|\left(T-z_{1} I\right) w_{n}\right\|+\left\|\left(T-z_{2} I\right)^{*} y_{n}\right\| .
\end{aligned}
$$

Therefore, $\left|\left(z_{1}-z_{2}\right)\left(w_{n}, y_{n}\right)\right| \rightarrow 0$ as $n \rightarrow \infty$. Since $z_{1} \neq z_{2},\left(w_{n}, y_{n}\right) \rightarrow 0$ as $n \rightarrow \infty$.

There exist complex numbers $a_{n}$ and $b_{n}$ and unit vectors $x_{n}$ in $H$ such that $w_{n}=a_{n} y_{n}+b_{n} x_{n},\left|a_{n}\right|^{2}+\left|b_{n}\right|^{2}=1$, and $\left(x_{n}, y_{n}\right)=0$. From the above paragraph we have that $a_{n} \rightarrow 0$, so $\left|b_{n}\right| \rightarrow 1$. Therefore, $\left\|\left(T-z_{1} I\right) x_{n}\right\| \rightarrow 0$ as $n \rightarrow \infty$.

Proof of Lemma 2. Let $\epsilon>0$. Since $a, b \in \sigma_{p}(T) \cap \partial W(T), a$ and $b$ are normal eigenvalues of $T$ [10, Theorem 2, p. 233]. Let $u, v \in H$ be unit vectors such that $T u=a u$ and $T v=b v$. Then $(u, v)=0$ and the closed subspace $N$ spanned by $\{u, v\}$ reduces $T$. Define $S \in B(H)$ as

$$
S u=\epsilon v, \quad S v=0, \quad S z=0 \text { for all } z \in N^{\perp} \text {. }
$$

Then we may write $T+S=A \oplus B$ corresponding to $H=N \oplus N^{\perp}$. Then the matrix representation of $A$ relative to $\{u, v\}$ is $A=\left[\begin{array}{cc}a & \epsilon \\ 0 & b\end{array}\right]$. Hence $\sigma(A)=\{a, b\} \subseteq \sigma(T)$. Clearly $\sigma(B) \subseteq \sigma(T)$. Therefore,

$$
\text { co } \sigma(T+S) \subseteq \operatorname{co} \sigma(T)=W(T) \text {. }
$$

Since $A$ is not a normal operator, $W(A)$ is the convex hull of a nondegenerate ellipse (i.e., not a straight line) with foci at $a$ and $b$ (see [1]). Since $W(A) \subseteq$ $W(T+S)$, we must have $\operatorname{co} \sigma(T+S) \neq W(T+S)$. Therefore, $T+S \notin \mathscr{L}$. Thus, since $\|S\|=\epsilon>0$ is arbitrary, $T \notin$ interior $\mathscr{L}$.

\section{BIBLIOGRAPHY}

1. William F. Donoghue, Jr., On the numerical range of a bounded operator, Michigan Math. J. 4 (1957), 261-263. MR 20 \#2622.

2. $\longrightarrow$, On a problem of Nieminen, Inst. Hautes Études Sci. Publ. Math. No. 16 (1963), 31-33. MR 27 \#2864.

3. N. Dunford and J. T. Schwartz, Linear operators. I: General theory, Pure and Appl. Math., vol. 7, Interscience, New York, 1958. MR 22 \#8302; II: Spectral theory. Selfadjoint operators in Hilbert space, Interscience, New York, 1963. MR 32 \#6181. 
4. N. Dunford, A survey of the theory of spectral operators, Bull. Amer. Math. Soc. 64 (1958), 217-274. MR 21 \#3616.

5. P. R. Halmos, Introduction to Hilbert space and the theory of spectral multiplicity, Chelsea, New York, 1957.

6. - Numerical ranges and normal dilations, Acta. Sci. Math. 25 (1964), 1-5.

MR 30 \#1399.

7. - A Hilbert space problem book, Van Nostrand, Princeton, N. J., 1967.

MR 34 \#8178.

8. S. Hildebrandt, The closure of the numerical range of an operator as spectral set, Comm. Pure Appl. Math. 17 (1964), 415-421. MR 29 \#3882.

9. - Numerischer Wertebereich und normale Dilatationen, Acta. Sci. Math.

(Szeged) 26 (1965), 187-190. MR 32 \#2906.

10。—, Über den numerischen Wertebereich eines Operators, Math. Ann. 163

(1966), 230-247. MR 34 \#613.

11. C. Meng, A condition that a normal operator have a closed numerical range, Proc. Amer. Math. Soc. 8 (1957), 85-88. MR 20 \#1223.

12. - On the numerical range of an operator, Proc. Amer. Math. Soc. 14 (1963),

167-171. MR 26 \#601.

13. G. H. Orland, On a class of operators, Proc. Amer. Math. Soc. 15 (1964), 75-79.

MR 28 \#480.

14. C. R. Putnam, On the spectra of semi-normal operators, Trans. Amer. Math. Soc.

119 (1965), 509-523. MR 32 \#2913.

15. - Commutation properties of Hibbert space operators and related topics,

Ergebnisse der Mathematik und ihrer Grenzgebiete, Band 36, Springer-Verlag, New York,

1967. MR 36 \#707.

16. - Eigenvalues and boundary spectra, Illinois J. Math. 12 (1968), 278-282.

MR 37 \#2030.

17. - The spectra of operators having resolvents of first-order growth, Trans.

Amer. Math. Soc. 133 (1968), 505-510. MR 37 \#4651.

18. F. Riesz and B. Sz.-Nagy, Leçons d'analyse fonctionnelle, Akad. Kiadó, Buda-

pest, 1952; English transl., Ungar, New York, 1955. MR 14, 286; MR 17, 175.

19. J. G. Stampfli, Hyponormal operators, Pacific J. Math. 12 (1962), 1453-1458.

MR $26 \# 6772$.

20. - Hyponormal operators and spectral density, Trans. Amer. Math. Soc. 117

(1965), 469-476. MR 30 \#3375.

21. - Analytic extensions and spectral localization, J. Math. Mech. 16 (1966),

287-296. MR 33 \#4687.

22. - Extreme points of the numerical range of a hyponormal operator, Michigan

Math. J. 13 (1966), 87-89. MR 32 \#4551.

23. Minimal range theorems for operators with thin spectra, Pacific J. Math.

23 (1967), 601-612. MR 37 \#4655.

24. - A local spectral theory for operators. II, Bull. Amer. Math. Soc. 75

(1969), 803-806. MR 39 \#6108.

25. - A local spectral theory for operators, J. Functional Anal. 4 (1969), 1-10.

MR 39 \#698.

26. A. Wintner, Zur Theorie der beschrankten Bilinearformen, Math. Z. 30 (1929),

$228-282$.

DEPARTMENT OF MATHEMATICS, IOWA STATE UNIVERSITY, AMES, IOWA 50010 